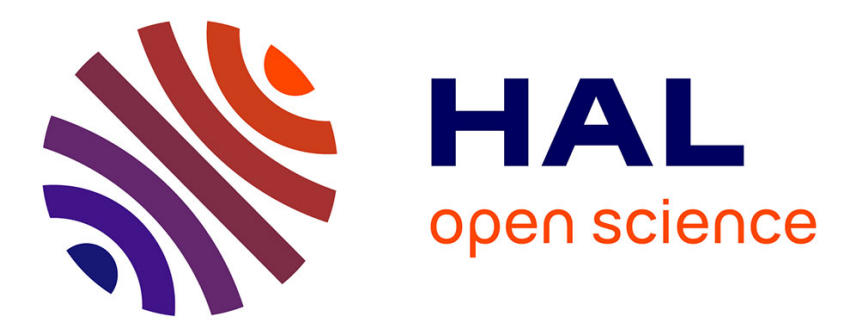

\title{
Multi-objective Human Resources Allocation in R\&D Projects Planning
}

Giacomo Galante, Antonella Certa, Mario Enea, Concetta Manuela La Fata

\section{To cite this version:}

Giacomo Galante, Antonella Certa, Mario Enea, Concetta Manuela La Fata. Multi-objective Human Resources Allocation in R\&D Projects Planning. International Journal of Production Research, 2009, 47 (13), pp.3503-3523. 10.1080/00207540701824233 . hal-00582319

\section{HAL Id: hal-00582319 https://hal.science/hal-00582319}

Submitted on 1 Apr 2011

HAL is a multi-disciplinary open access archive for the deposit and dissemination of scientific research documents, whether they are published or not. The documents may come from teaching and research institutions in France or abroad, or from public or private research centers.
L'archive ouverte pluridisciplinaire HAL, est destinée au dépôt et à la diffusion de documents scientifiques de niveau recherche, publiés ou non, émanant des établissements d'enseignement et de recherche français ou étrangers, des laboratoires publics ou privés. 


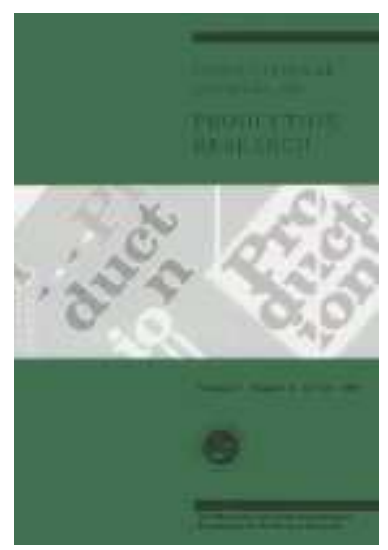

\section{Multi-objective Human Resources Allocation in R\&D Projects Planning}

\begin{tabular}{|c|c|}
\hline Journal: & International Journal of Production Research \\
\hline Manuscript ID: & TPRS-2007-IJPR-0559.R1 \\
\hline Manuscript Type: & Original Manuscript \\
\hline $\begin{array}{r}\text { Date Submitted by the } \\
\text { Author: }\end{array}$ & 13-Nov-2007 \\
\hline Complete List of Authors: & $\begin{array}{l}\text { Galante, Giacomo; University of Palermo, Dip.to di Tecnologia Mecc, } \\
\text { Prod e Ing Gestionale } \\
\text { Certa, Antonella } \\
\text { Enea, Mario } \\
\text { La Fata, Concetta }\end{array}$ \\
\hline Keywords: & PROJECT MANAGEMENT, DECISION SUPPORT SYSTEMS \\
\hline Keywords (user): & Human resources allocation, Mullti-objective optimization \\
\hline
\end{tabular}

\section{$\diamond$ ScholarONE" \\ Manuscript Central}




\title{
Multi-objective Human Resources Allocation in R\&D Projects Planning
}

\author{
Antonella Certa, Mario Enea, Giacomo Galante and Concetta Manuela La Fata
}

Dipartimento di Tecnologia Meccanica, Produzione e Ingegneria Gestionale

Università degli studi di Palermo

Italy

\begin{abstract}
In a R\&D department, several projects may have to be implemented simultaneously within a certain period of time by a limited number of human resources with diverse skills. This paper proposes an optimization model for the allocation of multi-skilled human resources to $R \& D$ projects, considering individual workers as entities having different knowledge, experience and ability. The model focuses on three fundamental aspects of human resources: the different skill levels, the learning process and the social relationships existing in working teams. The resolution approach for the multi-objective problem consists in two steps: firstly, a set of non-dominated solutions is obtained by exploring the optimal Pareto frontier and subsequently, based on further information, the ELECTRE III method is utilized to select the best compromise with regards to the considered objectives. The uncertainty associated to each solution is modelled by fuzzy numbers and used in establishing the threshold values of ELECTRE III, while the weights of the objectives are determined taking into account the influence that each objective has on the others.
\end{abstract}

Keywords: Human resources allocation, Multi-objective optimization, ELECTRE III, ANP.

\section{Introduction}

The assignment of human resources is a very important phase of the decision-making process, especially with respect to research and development $(R \& D)$ projects where performance strongly depends on human resources capabilities.

R\&D projects are an important function of any business' activity in particular for high-tech companies in electronics, software development, etc., whose primary function is product development and innovation. 
In a R\&D department, several projects may have to be implemented simultaneously within a certain period of time by a limited number of human resources with different skills, making the process of assigning tasks difficult. The purpose of this paper is to propose a multi-objective methodology to allocate human resources with different skill levels to projects that require several competencies. The model focuses on three fundamental aspects of human resources: the different skill levels, the learning process undertaken and the social relationships existing in working teams.

Regarding the first aspect, many studies show how it is possible to improve the efficiency of the assignment process by taking into account the resource capability level. Koshijima and Umeda (2001) formulated a method to allocate workers with different skill levels to tasks in order to minimize the project execution cost. Authors highlight how the traditional approach of project management doesn't give explicit consideration to human factors. Based on an equation representing the dynamic working process, the results show the improvement in the economic performance and in the project management process. The authors propose a linear programming model in which certain levels of skill are required to perform a task by multiple engineers who are classified on the basis of their experiences and capabilities.

Also, Gomar et al (2000) developed a linear programming model to optimize the assignment process of a partially multi-skilled workforce. Human resources have a range of skills that allow them to participate in one or more projects. In particular, the model considers four objective functions: minimizing the total number of workers used on a project, increasing employment on the construction phase, minimizing human resource hires and fires and minimizing the number of human resource switches between crews.

Bassett (2000) proposed a model to optimally assign people to roles in order to minimize outside contracts by better utilization of the available expertise. A one-month assignment is proposed and the scheduling problem is solved by mathematical programming and heuristic approaches.

Significant studies have examined the learning process, the second aspect considered herein, and stated that it has significant potential to improve productivity in organizations.

Wu and Sun (2006) developed a mixed nonlinear program for multi-project R\&D scheduling and staff allocation problems, which considers the staff learning effect. The goal is to minimize outsourcing costs, assuming the need to turn to outsourcing strategy whenever a task cannot be completed before its due date. A genetic algorithm (GA) is proposed to solve the problem. The learning process is also considered by Nembhard (2001), who suggested a heuristic approach for assigning workers to tasks based on the learning rate of the individual workers. The author shows by simulation that worker assignment based on empirical learning parameters has significant potential to increase the overall productivity. 
Some studies highlight the importance of the third aspect, the social relationships between workers. In particular, Yaakob and Kawata (1999) studied the problem of placement of workers in a production line. By using linguistic variables and triangular fuzzy numbers, they express the worker's suitability for each job. Since human resources operate in team, the relationships among workers are included as input data in order to make a more accurate decision. The team relationship function is calculated as the sum of all the relationships between pair of workers. The objective is the maximization of a composite function obtained by summing the workers suitability and the relationship among the team members for each job.

Shen et al (2002) also proposed a multi-criteria assessment model that considers the relationships between human resources. They underline the fact that several workers, with different skills and expertises, may share the same role in the organization but the selection of appropriate individuals, based merely on the relationship between a role and a task, is not very effective. They use linguistic variables and fuzzy numbers to evaluate worker suitability for each task. In particular, the task assignment considers three factors. First, the comparison between the workers' skill sets and the skills required to perform a task determines the worker capability in performing the task. The other two factors take into account the social relationships among workers and the relation among tasks, respectively, the latter being either conflicting or complementary. In order to prevent fraud, conflicting tasks must be performed by different workers. The authors also take into consideration the learning process to evaluate worker performance.

Extensive research has been done on handling the multi-objective human resource assignment. However, for the most part, the goal is to optimize a single utility function determined as the weighted sum of the score functions. The difficulty of normalizing the objective functions and of quantifying the weights, however, is well known.

In this paper, the search for the optimal solution is carried out in two steps. Firstly, a set of non-dominated solutions is obtained by exploring the optimal Pareto frontier and subsequently, based on further information, the decision maker can select the best compromise among the objectives, without a priori introduction of arbitrary weights.

Yoshimura et al (2006) developed a project selection algorithm for choosing the set of projects that maximizes the total estimated profit and then presented a human resources allocation algorithm for optimally placing human resources among the selected projects. A genetic algorithm is used to obtain a Pareto solution set from which the optimal solution is selected. The process for discarding unsuitable solutions is based on setting lower limit constraints for each objective considered; therefore the authors do not suggest any formalized tool for this phase of the optimization procedure. 
Conversely, the present work uses the ELECTRE III multi-criteria method as a tool to support the decision maker's process of choosing the optimal solution. By using proper thresholds, this method allows the decision maker to take into account the uncertainty and ambiguity that affect the performance evaluation.

The uncertainty in estimating some input parameters of the model is expressed by the use of fuzzy numbers, thus the value of each objective function corresponding to a certain solution is also a fuzzy number. These values are used to calculate the ELECTRE III thresholds. A set of weights for the decision criteria is introduced in order to apply ELECTRE III method. Its evaluation is difficult in our case since the decision criteria are not independent. In order to support the decision maker regarding this aspect, an adaptation of the Analytic Network Process (ANP) technique is proposed.

\section{Model description}

The problem here is to allocate the available human resources to a fixed number of projects that have to be carried out totally or partially within a certain scheduling period. The projects to be planned are those previously selected by the company according to the resources availability. In this context, no explicit mention to project's activities is made, but the competencies needed for their execution and the total time required for each competency are considered.

In R\&D projects, each human resource often participates in more than one project simultaneously and works in a team within a specific project. Furthermore, each activity is usually allowed to stop and then restart during project execution. Therefore, human resource flexibility in project implementation, at least in the planning phase considered here, can lead to ignoring or disregarding of existing relationships between activities.

The challenge here is not a classical resource-constrained project-scheduling problem (RCPSP), in which the objective is generally the minimization of project completion time, considering the precedence constraints among activities and the maximum availability of the resources. In RCPSP, the human resource allocation problem consists of determining the number of people assigned to each task. The resources are considered as uniform entities and their particular abilities and personalities are not taken into account.

On the contrary, the proposed model takes into account these factors explicitly and it aims to maximize the expected quality in project execution, allocating human resources to activities by considering their owns abilities and the importance that each skill brings to the project. In R\&D organizations, human resources are so fundamental that management must optimize their utilization. A success of a company can be enhanced when management encourages a positive and collaborative work environment, allowing workers to grow and mature. These two aspects are also considered in the model, which is actually a multi-objective problem. 
Described below are the objective functions that maximize the satisfaction of skills required by projects, the increase of skills and the human resource satisfaction in team working. For the sake of simplicity, the scheduling interval, whose duration usually depends on the environment in which companies work, is assumed unitary. The projects that need to be scheduled are just those beginning in this interval, while for those already started and not completed, it is assumed to maintain the previous planned resource assignments.

\subsection{Nomenclature}

I number of projects;

$J \quad$ human resources units;

$K \quad$ number of skills;

$c_{j k} \quad$ level of skill $k$ of the resource $j$ measured on $[0,1]$ range and evaluated at the beginning of the planning period;

$p_{k i} \quad$ relative importance of skill $k$ for project $i$;

$o_{k i} \quad$ time required by project $i$ for skill $k$ measured as a fraction of the planning period;

$a_{j, \max } \quad$ resource $j$ time availability measured as a fraction of the planning period;

$p_{i} \quad$ relative importance factor of project $i$;

$\beta_{j k} \quad$ learning curve parameter for resource $j$ and skill $k$;

$x_{j k i} \quad$ time that resource $j$ works on project $i$ dispensing skill $k$ measured as a fraction of the planning period;

$y_{j k i} \quad$ binary variable that takes value 1 if resource $j$ works on project $i$ dispensing skill $\mathrm{k}, 0$ otherwise;

$n_{j i} \quad$ total number of skills supplied by resource $j$ working on project $i$;

$H_{j i} \quad$ binary variable that takes value 1 if resource $j$ works on project $i, 0$ otherwise.

\subsection{Objectives functions}

The three objectives here considered are the maximization of the project quality, the increase of the resources skill and the satisfaction in working team. They are expressed by the following objective functions.

\subsubsection{Project quality}

Project quality mainly depends on the satisfaction of skills required by each project. Therefore the first objective function to maximize is: 


$$
F_{1}=\sum_{j, i, k} p_{i} \cdot c_{j k} \cdot p_{k i} \cdot x_{j k i}
$$

where:

$i=1, \ldots, I$ is the generic project among those in business portfolio $I$;

$j=1, \ldots, J$ represents the human resource among those available;

$k=1, \ldots, K$ is the generic skill among those $K$ needed to complete the project set $I$.

The decision variable $x_{j k i}$ is continuous and defined in [0,1] range. It represents a fraction of the unitary planning time that resource $j$ employs to bring the skill $k$ to the project $i$. Furthermore, every skill has a different importance in reaching the stated goals for each project, so a weight $p_{k i}$ is introduced to explain such priority. For every project, the sum of these weights is equal to 1 :

$$
\sum_{k} p_{k i}=1
$$

Each project is also characterized by a coefficient $p_{i}$ that expresses how the considered project is strategically important among all those in the business portfolio. Also in this case, the sum of the aforementioned coefficients is equal to 1 :

$$
\sum_{i} p_{i}=1
$$

The generic worker $j$ has an initial skill level $c_{j k}$ that represents, for each skill $k$, the worker's ability in performing those activities that require $k$. All parameters $c_{j k}$ are obtained from qualitative judgments expressed by a team of experts by using the procedure described in next section.

The objective function is subject to the following constraints:

$$
\sum_{k, i} x_{j k i} \leq a_{j, \max } \quad \forall j
$$

with

$$
x_{j k i} \geq 0 \quad \forall j, k, i
$$

and $a_{j, m a x}$ is the resource time availability within the planning period. Since this period is supposed to be unitary, the value of $a_{j, \max }$ is equal to 1 for workers not yet hired in projects already started and still being executed. On the contrary, the availability of human resources already hired is less than the planning period given that the engagements scheduled have to be carried out.

The respect of each skill $k$ required by the project $i$ is expressed by 


$$
\sum_{j} x_{j k i}=o_{k i} \quad \forall k, i
$$

where the $o_{k i}$ is the temporal request of skill $k$ by the project $i$.

Considering that it could be unproductive to employ a resource for only a small fraction of time, it is here supposed that each worker's employment must be not less than a fixed percentage $r_{k i}$ of demand $o_{k i}$ :

$$
\begin{aligned}
& x_{j k i} \geq\left(a+r_{k i} \cdot o_{k i}\right) \cdot y_{j k i} \quad \forall j, k, i \\
& x_{j k i} \leq y_{j k i}
\end{aligned}
$$

where $y_{j k i}$ is a Boolean variable equal to 1 if the human resource $j$ works on project $i$ dispensing skill $k$ and 0 otherwise. Moreover, the constraint assures that $y_{j k i}$ takes 1 if $x_{j k i}$ has a value not equal to 0 , becomes 0 if $x_{j k i}$ has a value equal to 0 . The parameter $a$ is a small arbitrary number that assures the respect of the previous condition when $o_{k i}$ is equal to 0 .

In order to focus the attention of each resource on few goals, it is assumed that the employer can at most participate in a stated number $N$ of projects.

The introduction of two dummy variables $n_{j i}$ and $H_{j i}$ is necessary to express this condition.

The first one, $n_{j i}$, is an integer variable representing the number of competencies totally performed by human resource $j$ in completing project $i$ :

$$
n_{j i}=\sum_{k} y_{j k i} \quad \forall j, i
$$

The second dummy variable, $H_{j i}$, is Boolean and takes 1 if resource $j$ works on project $i$, regardless of the specific competency, and 0 otherwise. The relationship between the variables just defined is expressed by:

$$
\begin{array}{ll}
n_{j i} \leq U_{b n} \cdot H_{j i} & \forall j, i \\
H_{j i} \leq n_{j i} &
\end{array}
$$

where $U_{b n}$ is the upper bound of $n_{j i}$ stated as the number of skills required by each project.

Constraint (9) implies that $H_{j i}$ is 0 if $n_{j i}$ is 0 and 1 if $n_{j i}$ is not equal to zero.

It is now possible to express the constraint concerning the maximum number of projects on which the generic resource $j$ can work as:

$$
\sum_{i} H_{j i} \leq N
$$

For a given skill $k$, in order to avoid an over splitting of workloads between resources employed on the same project, it is convenient to fix the maximum number of team members. In this context, considering that $R \& D$ projects are taken into account, the number of team members is at most 2. 
Therefore:

$$
\sum_{j} y_{j k i} \leq 2 \quad \forall k, i
$$

\subsubsection{Increase of the resources skill}

The second objective function aims to maximize the minimum amount of skill available for business activities by learning process. For each skill $k$, the total availability is calculated by summing the amount of the same skill globally held by all resources.

The initial value $c_{j k}$ arises from the last evaluation made by a team of experts stating their judgments by linguistic variables connected to fuzzy numbers, as described in section 3 . The value of $c_{j k}$ is the crisp value obtained by defuzzificating the corresponding fuzzy number.

To evaluate the resource skill improvement, the following learning curve is used:

$$
f(t)=1-e^{-\beta_{j k} t}
$$

where $\beta_{j k}$ is the specific parameter for resource $j$ and skill $k$ and $t$ is the learning time.

In order to simplify the model, the improvement is calculated by a linear approximation of the learning curve. A growth factor $m_{j k}$ is calculated as the derivative of the learning curve in correspondence to $c_{j k}$ and the improvement is therefore expressed by:

$$
\left(m_{j k} \cdot \sum_{i} x_{j k i}\right)
$$

The linear approximation is valid because the scheduling period is significantly smaller than the one in which the learning curve is defined.

Resource ability at the end of the planning period is therefore:

$$
c_{j k}+m_{j k} \cdot \sum_{i} x_{j k i}
$$

while the generic skill $k$ globally available at the end of same period is:

$$
c_{k}=\sum_{j}\left(c_{j k}+m_{j k} \cdot \sum_{i} x_{j k i}\right)
$$

The second objective function is to maximize the minimum value of skill availability:

$$
F_{2}=\min _{k} c_{k}
$$

\subsubsection{Satisfaction in work team}

A function that takes into account the preference expressed by resources $j$ and $l$ in working together within the same team is here formulated. This preference is defined by a coefficient $\alpha_{j l}$ in the so-called social 
relationships matrix built on the base of judgments expressed by human resources. These judgments are linguistic variables, which are linked to fuzzy numbers. The social relationships matrix is updated after each collaboration teamwork experience.

Therefore, the objective function to maximize is:

$$
F_{3}=\sum_{j, l, k, i} \alpha_{j l} \cdot y_{j k i} \cdot y_{l k i}
$$

\section{Evaluation of $c_{j k}$ and $\alpha_{j l}$ parameters}

The judgments about the individual skill level and the affinity grade of each couple are expressed by linguistic variables.

Zadeh (1975) proposed a fuzzy approach to numerically represent the uncertainty associated to qualitative evaluations. Fuzzy numbers are standard fuzzy sets defined on a set of real numbers. In this paper, triangular and trapezoidal fuzzy numbers are used. Triangular fuzzy numbers are widely used due to their simplicity and solid theoretical basis (Pedrycz, 1994).

The membership function of a triangular fuzzy number $A$ is $\mu_{A}: \mathrm{R} \rightarrow[0,1]$ and can be represented by the equations:

where $d<m<u$.

$$
\mu_{A}(x)= \begin{cases}\frac{x-d}{m-d} & \text { when } x \in[d, m] \\ \frac{u-x}{u-m} & \text { when } \mathrm{x} \in[m, u] \\ 0 & \text { otherwise }\end{cases}
$$

Consequently, a triangular fuzzy number is fully characterized by three real numbers $(d, m, u)$. The parameter $m$ gives the maximum grade of $\mu_{A}(x)$ that is equal to $1 ; d$ and $u$ are the lower and upper bounds of the definition interval.

Analogously, the membership function of a trapezoidal fuzzy number $B$ is $\mu_{\mathrm{B}}: \mathrm{R} \rightarrow[0,1]$ and can be represented by the equations: 


$$
\mu_{B}(x)=\left\{\begin{array}{cl}
\frac{x-d}{m-d} & \text { when } x \in[d, m] \\
1 & \text { when } x \in[m, n] \\
\frac{u-x}{u-n} & \text { when } x \in[n, u] \\
0 & \text { otherwise }
\end{array}\right.
$$

where $d<m<n<u$.

Similarly, a trapezoidal fuzzy number is fully characterized by four real numbers $(d, m, n, u)$. The parameters $m$ and $n$ give the maximum grade of $\mu_{B}(x)$.

Figure 1 shows the linguistic terms used to express the judgments about $c_{j k}$ and $\alpha_{j l}$ and the corresponding fuzzy numbers.

The MIN fuzzy operator is used in this context in order to aggregate the judgments expressed by each expert, so $\alpha_{j l}$ represents the minimum affinity level declared from each pair of resources. Concerning the evaluation of $c_{j k}$, the same operator is applied because the procedure guarantees that the estimated skill level is really transferred to the project each resource works on.

The values of $c_{j k}$ and $\alpha_{j l}$ in the objective functions are the crisp values obtained by defuzzificating the corresponding fuzzy number using the centroid method. The information about the uncertainty of the parameters will be used in the second step of the optimization procedure (section 4.2.2).

\section{Insert Fig.1}

\section{Resolution approach}

As mentioned in the introduction, the resolution approach proposed for the multi-objective optimization problem consists of two steps. In the first, various trade-off solutions best approximating the Pareto-optimal frontier are determined. Next, in order to select the optimal solution among those obtained in the previous step, the ELECTRE III multi-criteria method is applied. Further information about the problem is then used to compare the solutions. 


\subsection{First step}

In this phase the solutions that best describe the Pareto optimal frontier are individuated and the number of those to be considered in the next step of selection process is reduced, by eliminating similar solutions . In this way, maintaining a complete description of the non-dominated solution space, the selection procedure is made easier.

The Lexicographic Goal Programming Method (LGP), the Weighted Sum Method and the $\varepsilon$-constraint Method are used to determine those solutions. To reduce the number of solutions being submitted to ELECTRE III and maintain a complete description of the Pareto-optimal frontier, the concept of crowding distance is then applied.

\subsubsection{Pareto-optimal solutions}

The LGP is initially used to individuate the extreme Pareto-optimal solutions. This method considers the three objective functions separately, thereby reducing a multi-objective problem to a mono-objective one. For example, these sequential steps determine one extreme solution:

- maximizing $F_{1}$ as a single objective problem;

- maximizing $\mathrm{F}_{2}$ considering the previous value of $\mathrm{F}_{1}$ as a further constraint;

- maximizing $\mathrm{F}_{3}$ considering the previous values of $F_{1}$ and $F_{2}$ as further constraints.

The procedure is analogously applied changing the objectives hierarchy to find the other five solutions, normalized using the expression:

$$
F_{i, n o r m}=\frac{F_{i}-F_{i}^{\min }}{F_{i}^{\max }-F_{i}^{\min }} \quad(i=1,2,3)
$$

The next step is to apply the Weighted Sum Method to reduce the objectives set into a single objective by multiplying each objective function by a weight, resulting in the following composite objective function:

$$
F=w_{1} \cdot F_{1, \text { norm }}+w_{2} \cdot F_{2, \text { norm }}+w_{3} \cdot F_{3, \text { norm }}
$$

with:

$$
\sum_{i=1}^{3} w_{i}=1
$$

A description of the Pareto-frontier is obtained by varying the weights and solving the problem: 
$\max F$

subject to constraints (4), (5), (6), (7), (8), (9), (10) and (11).

Since our model is non-linear, the solution space may be non-convex and the Weighted Sum Approach (Deb, 2001) may not be able to determine all Pareto-optimal solutions.

Anyway, ignoring the shape of the objective space, the setting of the weights vector to obtain a complete description of the optimal Pareto frontier is really difficult: a uniformly distributed set of weights vectors does not correspond to a uniformly distributed set of Pareto-optimal solutions. Moreover, different weights vectors do not necessarily lead to different Pareto-optimal solutions. For these reasons, in order to find other solutions in the region where few solutions have been found by using the Weighted Sum Approach, the $\varepsilon$-constraint Method is applied. This method is able to find solutions also in the non-convex region. As Haimes et al. (1971) suggested, the problem can be modified keeping one of the objectives and restricting the others within user-specified values.

The problem becomes:

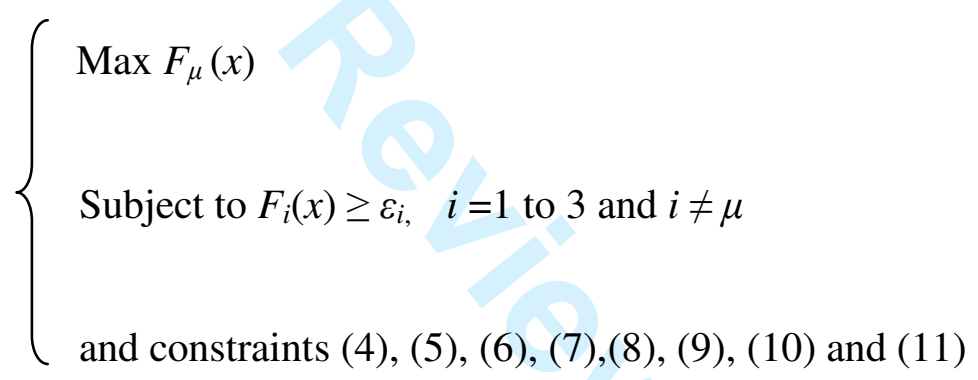

Hence, different Pareto-optimal solutions can be found by using different sets of $\varepsilon_{i}$ that must be chosen within the minimum and maximum values of the objective functions found by using the LGP. The choice of a particular set of values for $\varepsilon_{i}$ restricts the possible location of the solution to a desired region of the Paretooptimal frontier.

\subsubsection{Reduction of the number of solutions}

This procedure reduces the solutions obtained in the previous step by ranking them on the basis of the crowding distance. The crowding distance provides an estimate of the density of solutions around each solution belonging to the frontier. Considering a generic objective $i$, the $n$ solutions are sorted in descending order. Fixing $d_{i}{ }^{1}=d_{i}{ }^{n}=\infty$, the crowding distance of the generic solution $z$ is expressed by:

$$
d_{i}^{z}=\frac{F_{i}^{z+1}-F_{i}^{z-1}}{F_{i}^{\max }-F_{i}^{\min }} \quad(i=1,2,3 ; z=2, \ldots, n-1)
$$


where $F_{i}^{\max }$ and $F_{i}^{\text {min }}$ are the maximum and minimum values of the objective function $i$ of the solutions belonging to the frontier. The overall crowding distance of the solution $z$, considering all objectives, is:

$$
d^{z}=\sum_{i=1}^{3} d_{i}^{z} \quad(z=2, \ldots, n-1)
$$

The solutions selected for the next step are the first $m$, with the higher values of the parameter.

\subsection{Second step: ELECTRE III}

ELECTRE III (Roy, 1996) is a multi-criteria decision making procedure that can be applied when a set of alternatives must be ranked according to a set of criteria reflecting the decision maker's preferences. In a multi-criteria decision problem, although logical and mathematical conditions required to determine an optimum solution do not exist, a solution representing a good compromise according to the conflicting criteria established can be individuated. The ELECTRE III method is based upon pseudo-criteria. Using proper thresholds, a pseudo-criterion takes into account the uncertainty and ambiguity that can affect the evaluation of the performance, so that, if the difference in the performance of two alternatives is minimal, according to a certain criterion, such alternatives can be considered indifferent according to that criterion. Another peculiarity that differentiates ELECTRE III from other methodologies is that it is not compensative, which means that a very bad score in one objective function is not compensated by good scores in other objectives. In other words, the decision maker will not choose an alternative if it is very bad compared to another one, even on a single criterion. This occurs if the difference between the values of an attribute of two alternatives is greater than a fixed veto threshold. ELECTRE III is based upon outranking relations: an alternative outranks another alternative if sufficient reasons exist to assert that the first is as good as the second and good reasons to reject such assertions do not exist. Outranking is therefore based upon a concordance/discordance principle. This principle consists of the verification of the existence of a concordance of criteria in favour of the assertion that one alternative is as good as another and that a verifiably strong discordance among the score values that may reject the previous assertion does not exist.

The following thresholds are introduced:

$q$ - indifference threshold;

$p$ - preference threshold;

$v$ - veto threshold;

where $q \leq p \leq v$. 
Therefore, the decision maker is involved to supply his/her preferences by choosing the objective function weights and the indifference, preference and veto thresholds for each objective function.

\subsubsection{Objective weights}

The weights associated to each objective function represent the relative importance of each goal compared to the others. In this case, the objectives are not independent: reaching one goal positively affects the other goals. Since it is impossible to translate these influences between goals into mathematical expressions, the model does not take them into account.

In particular, let $\mathrm{Ob}_{1}, \mathrm{Ob}_{2}$ and $\mathrm{Ob}_{3}$ represent the three goals: project quality, increase of the resources skill level and satisfaction in working teams respectively. The following relationships may be considered:

- $\mathrm{Ob}_{1} \rightarrow \mathrm{Ob}_{3}$ : a good development of project implies an improvement in social relations between human resources who work together;

- $\mathrm{Ob}_{3} \rightarrow \mathrm{Ob}_{1}$ : a good affinity among resources working together positively influences project development, since it puts the resources in a position to entirely bring their own skills to the project;

- $\mathrm{Ob}_{1} \rightarrow \mathrm{Ob}_{2}$ : a good development of project implies a faster learning process;

- $\mathrm{Ob}_{2} \rightarrow \mathrm{Ob}_{1}$ : improvement in skill level during project execution implies improvement in expected project quality;

- $\mathrm{Ob}_{2} \rightarrow \mathrm{Ob}_{3}$ : the satisfaction for the individual skill level increase implies an improvement of teamwork relationship;

- $\mathrm{Ob}_{3} \rightarrow \mathrm{Ob}_{2}$ : a higher level of teamwork relations leads to more professional growth in human resources.

The Analytic Network Process (ANP) is a valid and useful approach in selecting a particular solution among all those found when the business objectives are not independent (Saaty, 1996). The ANP method, as the more famous AHP, is based on the comparison between couple of alternatives, so it may handle only a restricted number of alternatives. Saaty has shown that to maintain a reasonable consistency when deriving priorities from paired comparisons, the number of elements being considered must be less or equal to nine (Saaty, 1980).

In our case, a complete description of the Pareto optimal frontier is presented to the decision maker, implying a number of solutions that makes ANP unusable. For this reason and for the ones mentioned in section 4.2, the ELECTRE III method is chosen in order to select the best solution. However, the dependence among the objectives cannot be disregarded and here is considered in determining the weights. Such dependence implies 
a modification of the objectives importance based only on the fulfilment of the company expectations. For example, as already mentioned before, a good affinity among resources in working team positively influences the expected project quality and therefore the importance of objective 3 increases.

For such reasons, an adaptation of the Analytic Network Process (ANP) to the particular considered problem is proposed.

The ANP extends the hierarchy structure of the AHP method to a network structure, whose nodes consist of clusters of elements (criteria, alternatives, etc.) and arcs represent the link among them. In particular, ANP deals with the dependence of elements within a cluster (inner dependence) and among elements belonging to different clusters (outer dependence). The influence that a set of elements belonging to the same cluster has on each element of the problem is represented by a priority vector, obtained by paired comparisons in the usual way of AHP. These priority vectors are grouped and arranged into a matrix called unweighted supermatrix.

The ANP calculation process employs other two matrices, namely the weighted supermatrix and the limit supermatrix. The weighted supermatrix is obtained by multiplying all the elements of the unweighted supermatrix by the corresponding cluster weight. The limit supermatrix is obtained by multiplying the weighted supermatrix by itself until the values in each row are equal. These values constitute the final priority of the elements. The simple network structure of our decisional problem is represented in figure 2.

\section{Insert Fig.2}

The loop in figure 2 represents the inner dependence. Therefore, it is possible to calculate the relative importance of each criterion with respect to company's satisfaction and the influence that each one has on the others. The relative importance of the objectives is the weight introduced in ELECTRE III. 


\subsubsection{Thresholds.}

The indifference threshold $q$ is the minimum difference between values taken from a goal that the decision maker considers meaningful to state that one solution is preferable over another. It is due to a real indifference of the decision maker about two similar solutions but it should take into account the uncertainty associated to each solution. Since available information is usually not sufficient, the last issue is not properly taken into account.

In this study, the indifference threshold is associated to the uncertainty described by the fuzzy number corresponding to the generic objective function calculated in a solution. It is useful to keep in mind that judgements about skill levels and affinity are the fuzzy numbers $c_{j k}$ and $\alpha_{j k}$.

To solve the model described in section 2, these numbers have been defuzzified using the Centroid Method to find the corresponding crisp value. Nevertheless, once the model is solved, it is possible to express the value of each objective function by a fuzzy number obtained executing the operations on the fuzzy number of the correspondent function. For a given goal and solution, the indifference threshold is stated as the difference between the upper bound $u$ of the defined interval and the central value $m$ of the fuzzy number. If the crisp value of the objective function of a solution is greater than the corresponding value of another solution but this gap is lower than the interval threshold, this difference could be due to the uncertainty rather to an effective prevalence of the first solution on the second. The preference threshold $p$ represents, instead, the minimum gap between values taken by the objective function which the decision maker thinks as important to strictly state that a solution is preferable over another one.

A deep knowledge of the problem is required to fix $p$; the only possible comment is that $p$ is significantly greater than $q$.

Finally, if an alternative is worse than another based on a certain criterion for a value greater than the so called veto threshold $v$ and the same solution is strictly better based on other criteria, this solution is declared outranked by the other. Using ELECTRE III, it is not necessary to introduce the veto threshold. In this study, it is used only for the first objective function, as showed in the numerical example in the following section.

\section{Numerical example}

The proposed approach has been applied to a simulated case including 8 human resources, 5 projects and 5 skills. The input data are reported in Tables 1-7.

The data are generated on the basis of the following considerations to make the model as realistic as possible:

- the sum of time required in Table 1 matches the constraint on maximum availability of the resources; 
- each resource has unevenly distributed skill levels;

- a value of $o_{i k}=0$ implies $p_{i k}=0$;

- a judgment value smaller than "low" (see figure 1) about $c_{j k}$ implies that no possibility of growth is given to resource $j$ with relation to skill $k$;

- the values of $\beta_{k}$ have been created assuming that the skill level improvement, from a minimum value of 0.3 (centroid of the fuzzy number associated with the linguistic variable "low") to a maximum value of 0.99 , takes place in 5-10 times the scheduling period, randomly generated .

For the sake of simplicity, it is assumed that, for each competence, all resources are on the same learning path during the project's execution. thus table 6 shows $\beta_{k}$ and not $\beta_{j k}$. It is thus considered a learning curve for each skill, regardless of the particular human resource taken into account.

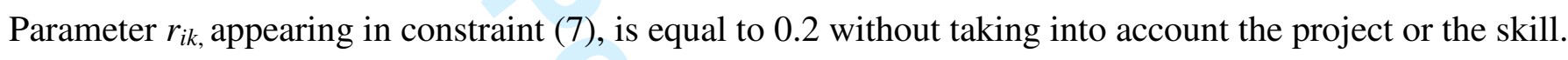

Each worker may works on a number $N$ of projects, which are at most 3.

\section{Table 1}

Table 2

Table 3

Table 4

Table 5

Table 6

Table 7

By using the procedure described in section 4.1.1 and Lingo 10 software, 50 solutions are found. Among those, 15 solutions are selected by the procedure described in section 4.1.2 (see table 8). The second column of the table below shows the method used to find the solution.

\section{Table 8}

As highlighted in section 4.2.1, the weights associated to the objective functions and required by ELECTRE III are found using the ANP method and Super Decisions Software. 
Table 10 shows the comparison between couples of objectives with respect to the company satisfaction, while table 11, 12 and 13 represent the evaluation of mutual dependences. The pair wise comparisons are expressed by Saaty's semantics scale (table 9).

\section{Table 9}

Table10

Table 11

Table 12

Table 13

Since the network is characterized by one cluster, the unweighted supermatrix in table 14 matches the weighted one. In table 15 the global weights of the limit matrix are reported.

\section{Table 14}

Table 15

To evaluate the indifference threshold, the procedure described in 4.2 .2 is applied. Figure 3 shows, as example, the fuzzy number that matches the first objective function calculated in solution 11 by using equation (1). The corresponding threshold is the difference between the upper bound $u$ of the definition interval and the central value $m$.

\section{Fig.3}

For the sake of simplicity, the preference threshold is fixed at twice the indifference threshold. The veto threshold, used for the first objective only, is three times the indifference threshold.

The indifference thresholds for each objective function and for each solution are in table 16.

\section{Table 16}

Applying ELECTRE III to alternatives in table 8, normalizing the values of the objective functions by equation (20), solutions 9 and 10 are selected as the best compromise among those supplied. Since ELECTRE 
III has not been able to select between these two solutions, the choose of the solution to implement has to be carried out by the decision maker on the basis of further aspects not considered in the present model.

Tables 17 and 18 show the workers assigned to the projects and the skills dispensed for the two selected solutions.

\section{Table 17}

\section{Table 18}

Tables 17 and 18 highlight that worker 7 is not involved during the considered planning period, that confirms the accuracy of the results obtained. In fact, since the global resources availability is greater than the request needed in projects execution, the most unsuitable resource in matching the business goals is not hired. In particular, looking at table 7, it is evident that the resource 7 has the lowest affinity with the others and so he/she is penalized in hiring.

\section{Conclusions}

The developed multi-objectives model allows to find the best allocation of human resources to projects considering worker features. The resolution approach of the problem consists in two steps: first, a set of nondominated solutions that describes the optimal Pareto frontier is obtained and subsequently, based on further information, the ELECTRE III method is used to select the solution that represents the best compromise according to the considered objectives. The advantages in applying the described procedure compared to the classical one, which optimizes a utility function, are already aforementioned. Two further aspects of this method have to be here highlighted as innovative: the computation of the indifference threshold and the specification of the weights utilized for the ELECTRE III. The choice of the indifference threshold is subjective: it depends on the decision maker who expresses the equivalence judgment between two generic solutions according to a specific goal. However, in the problem handled, the most important aspect that needs to be considered is the uncertainty of values taken by the objective functions. In this paper a fuzzy approach is used to numerically translate the qualitative judgments of the different aspects of human resources. The uncertainty described by the fuzzy number corresponding to the generic objective function is used as value of the indifference threshold. Regarding the second aspect, the AHP method is usually utilized in literature to calculate the weights associated to each goal. In evaluating the relative importance between two objectives, it is hard for the decision maker to take into account the dependences between them. In order to overcome this 
problem, a modified version of ANP method is proposed. The ANP is generally used in alternatives selection phase and it is capable of handling interdependence among elements. Instead, in this specific case, the aim is not the choose of the best alternative, carried out by ELECTRE III, but the calculation of the weights of the objectives considered as elements belonging to a cluster.

Further developments can focus on the exploration of the Pareto frontier that could be improved by using a costrained multi-objective genetic algorithm. 


\section{References}

- Bassett, M., Assigning projects to optimize the utilization of employees' time and expertise, Computers \& Chemical Engineering, 2000, 24, 1013-1021.

- Deb, K., Multi-Objective Optimization Using Evolutionary Algorithms, John Wiley \& Sons, New York, 2001.

- Gomar, J., E., Terrien, R., K., Haas, C., T., Morton, D., P., Tucker, R., L., Assignment and allocation optimization of a partially multiskilled workforce, Internal Report of Center for Construction Industry Studies, University of Texas at Austin, 2000.

- Haimes, Y., Y., Lasdon, L. S., Wismer, D., A., On a bicriterion formulation of the problems of integrated system identification and system optimization, IEEE Transactions on Systems, Man and Cybernetics, 1971, 1(3), 296-297.

- Koshijima, I., Umeda, T., Human Resource Allocation in Project Management - Management Science Approach, Internal report of Chiba Institute of Technology Tsudanuma, Narashino, Chiba, 2001.

- Nembhard, D., A., Heuristic approach for assigning workers to tasks based on individual learning rates, International Journal of Production Research, 2001, 39(9), 1955-1968.

- Pedrycz, W., Why Triangular Membership Functions?, Fuzzy Sets and Systems, 1994, 64(1), 21-30.

- $\quad$ Roy, B., Multicriteria methodology for decision aiding, Kluwer, Dordrecht, The Netherlands, 1996.

- Saaty, T., L., Decision making with dependence and feedback: The analytic network process, RWS Publications, Pittsburgh, 1996.

- $\quad$ Saaty, T.L., The Analytic Hierarchy Process, N.Y., McGraw Hill, New York, 1980.

- Shen, M., Tzeng, G.-H., Liu, D.-R., Multi-criteria task assignment in workflow management systems, Proceedings of the 36th Hawaii International Conference on System Sciences, Big Island, Hawaii, USA, January 6-9 2003, 202-210 .

- Wu, M-C., Sun S-H., A project scheduling and staff assignment model considering learning effect, International Journal of Advanced Manufacturing Technology, 2006, 28, 1190-1195.

- Yaakob, S., B., Kawata, S., Workers'placement in an industrial environment, Fuzzy sets and system, 1999, 106 (3), 289-297.

- Yoshimura, M., Fusimi, Y., Izui, K., Nishiwaki, S., Decision-making support system for human resource allocation in product development projects, International journal of Production Research, 2006, 44 (5), 831-848. 
- Zadeh, L., A., The Concept of a Linguistic Variable and Its Application to Approximate Reasoning, Information Sciences, 1975, 8(3), 199-249.

1

2

3

4

5

6

7

8

9

10

11

12

13

14

15

16

17

18

19

20

21

22

23

24

25

26

27

28

29

30

31

32

33

34

35

36

37

38

39

40

41

42

43

44

45

46

47

48

49

50

51

52

53

54

55

56

57

58

59

60 
Table 1 . Time required by any project for each skill $o_{k i}$

\begin{tabular}{c|ccccc}
\hline skill & 1 & 2 & 3 & 4 & 5 \\
\hline 1 & & & & & \\
2 & 0.25 & 0.4 & 0 & 0.15 & 0 \\
3 & 0 & 0.1 & 0.35 & 0.25 & 0.15 \\
4 & 0.25 & 0 & 0.1 & 0.35 & 0.2 \\
5 & 0.2 & 0.15 & 0.15 & 0.08 & 0.3 \\
\hline
\end{tabular}

Table 2. Time resource availability $a_{\text {i.max }}$

\begin{tabular}{ccccccccc}
\hline resource & 1 & 2 & 3 & 4 & 5 & 6 & 7 & 8 \\
\hline & 0.8 & 1 & 1 & 1 & 0.7 & 1 & 1 & 1 \\
\hline
\end{tabular}

Table 3. Relative importance factor of project $p_{i}$

\begin{tabular}{cccccc}
\hline project & 1 & 2 & 3 & 4 & 5 \\
\hline & 0.15 & 0.25 & 0.2 & 0.1 & 0.3 \\
\hline
\end{tabular}

Table 4. Relative importance of skill $k$ for project $i$

\begin{tabular}{c|ccccc}
\hline project & 1 & 2 & 3 & 4 & 5 \\
\hline 1 & & & & & \\
2 & 0.25 & 0.4 & 0 & 0.35 & 0 \\
3 & 0 & 0.3 & 0.15 & 0.5 & 0.05 \\
4 & 0.2 & 0 & 0.1 & 0.45 & 0.25 \\
5 & 0.1 & 0.35 & 0.15 & 0.15 & 0.25 \\
\hline
\end{tabular}

Table 5. Resource skill level $c_{i k}$

\begin{tabular}{c|ccccc}
\hline & \multicolumn{5}{c}{ skill } \\
resource & 1 & 2 & 3 & 4 & 5 \\
\hline 1 & 0.3 & 0.5 & 0.4 & 0.077 & 0.8 \\
2 & 0.4 & 0.7 & 0.077 & 0.8 & 0.3 \\
3 & 0.8 & 0.077 & 0.5 & 0.5 & 0.4 \\
4 & 0.4 & 0.5 & 0.7 & 0.6 & 0.077 \\
5 & 0.077 & 0.8 & 0.3 & 0.6 & 0.3 \\
6 & 0.4 & 0.3 & 0.6 & 0.077 & 0.8 \\
7 & 0.4 & 0.077 & 0.6 & 0.3 & 0.7 \\
8 & 0.8 & 0.3 & 0.077 & 0.5 & 0.4 \\
\hline
\end{tabular}

Table 6. Learning curve parameter $\beta_{k}$

\begin{tabular}{cccccc}
\hline skill & 1 & 2 & 3 & 4 & 5 \\
\hline & 0.7 & 0.75 & 0.55 & 0.5 & 0.65 \\
\hline
\end{tabular}


Table 7. Social relationships matrix $\alpha_{j l}$

\begin{tabular}{|c|c|c|c|c|c|c|c|c|}
\hline resource & 1 & 2 & 3 & 4 & 5 & 6 & 7 & 8 \\
\hline 1 & & 0.2 & 0.8 & 0.077 & 0.6 & 0.2 & 0.077 & 0.6 \\
\hline 2 & & & 0.8 & 0.6 & 0.077 & 0.2 & 0.4 & 0 \\
\hline 3 & & & & 0.6 & 0.2 & 0.8 & 0.4 & 0.2 \\
\hline 4 & & & & & 0.8 & 0.4 & 0.077 & 0.6 \\
\hline 5 & & & & & & 0.2 & 0.6 & 0.2 \\
\hline 6 & & & & & & & 0.077 & 0.6 \\
\hline 7 & & & & & & & & 0.077 \\
\hline 8 & & & & & & & & \\
\hline
\end{tabular}

Table 8. Pareto solutions to submit to ELECTRE III

\begin{tabular}{ccccc}
\hline Solution & Method used & $F_{1}$ & $F_{2}$ & $F_{3}$ \\
\hline 1 & l.g.p. & 0.156 & 3.383 & 2.000 \\
2 & l.g.p. & 0.139 & 3.383 & 11.800 \\
3 & l.g.p. & 0.151 & 3.228 & 16.000 \\
4 & l.g.p. & 0.132 & 3.321 & 16.000 \\
5 & l.g.p. & 0.181 & 3.198 & 1.000 \\
6 & $\varepsilon$ & 0.146 & 3.349 & 15.200 \\
7 & w & 0.143 & 3.377 & 13.800 \\
8 & w & 0.154 & 3.374 & 10.400 \\
9 & $\varepsilon$ & 0.150 & 3.349 & 14.400 \\
10 & w & 0.162 & 3.310 & 13.200 \\
11 & w & 0.137 & 3.357 & 15.600 \\
12 & w & 0.138 & 3.364 & 15.200 \\
13 & $\varepsilon$ & 0.176 & 3.265 & 4.400 \\
14 & $\varepsilon$ & 0.175 & 3.214 & 9.600 \\
15 & w & 0.179 & 3.242 & 4.400 \\
\hline
\end{tabular}

Table 9. Saaty Scale

\begin{tabular}{|c|c|c|}
\hline $\begin{array}{l}\text { Numerical } \\
\text { values }\end{array}$ & Verbal scale & Explanation \\
\hline 1 & $\begin{array}{l}\text { Equal importance of } \\
\text { both elements }\end{array}$ & $\begin{array}{c}\text { Two elements } \\
\text { contribute equally }\end{array}$ \\
\hline 3 & $\begin{array}{l}\text { Moderate importance of } \\
\text { one element over another }\end{array}$ & $\begin{array}{l}\text { Experience and judgment favour } \\
\text { one element over another }\end{array}$ \\
\hline 5 & $\begin{array}{l}\text { Strong importance of } \\
\text { one element over another }\end{array}$ & $\begin{array}{c}\text { An element is } \\
\text { strongly favoured }\end{array}$ \\
\hline 7 & $\begin{array}{l}\text { Very strong importance of } \\
\text { one element over another }\end{array}$ & $\begin{array}{c}\text { An element is } \\
\text { very strongly dominant }\end{array}$ \\
\hline 9 & $\begin{array}{l}\text { Extreme importance of } \\
\text { one element over another }\end{array}$ & $\begin{array}{c}\text { An element is favoured by at least } \\
\text { an order of magnitude }\end{array}$ \\
\hline $2,4,6,8$ & Intermediate values & $\begin{array}{c}\text { Used to compromise } \\
\text { between two judgments }\end{array}$ \\
\hline
\end{tabular}


Table10. Pair wise comparisons between objectives with respect to the company satisfaction

\begin{tabular}{c|ccc}
\hline $\begin{array}{c}\text { Company } \\
\text { Satisfaction }\end{array}$ & $\mathrm{Ob}_{1}$ & $\mathrm{Ob}_{2}$ & $\mathrm{Ob}_{3}$ \\
\hline $\mathrm{Ob}_{1}$ & 1 & 4 & 6
\end{tabular}

Table 11. Pair wise comparisons between objectives with respect to $\mathrm{Ob}_{1}$

\begin{tabular}{c|cc}
\hline $\mathrm{Ob}_{1}$ & $\mathrm{Ob}_{2}$ & $\mathrm{Ob}_{3}$ \\
\hline $\mathrm{Ob}_{2}$ & 1 & 2 \\
$\mathrm{Ob}_{3}$ & $1 / 2$ & 1 \\
\hline
\end{tabular}

Table 12. Pair wise comparisons between objectives with respect to $\mathrm{Ob}_{2}$

\begin{tabular}{c|cc}
\hline $\mathrm{Ob}_{2}$ & $\mathrm{Ob}_{1}$ & $\mathrm{Ob}_{3}$ \\
\hline $\mathrm{Ob}_{1}$ & 1 & 2 \\
$\mathrm{Ob}_{3}$ & $1 / 2$ & 1 \\
\hline
\end{tabular}

Table 13. Pair wise comparisons between objectives with respect to $\mathrm{Ob}_{3}$

\begin{tabular}{c|cc}
\hline $\mathrm{Ob}_{3}$ & $\mathrm{Ob}_{1}$ & $\mathrm{Ob}_{2}$ \\
\hline $\mathrm{Ob}_{1}$ & 1 & 3 \\
$\mathrm{Ob}_{2}$ & $1 / 3$ & 1 \\
\hline
\end{tabular}

Table 14. Unweighthed supermatrix

\begin{tabular}{cccccc}
\hline & & \multicolumn{2}{c}{ goal } & \multicolumn{3}{c}{ project objectives } \\
\cline { 3 - 6 } Cluster node labels & company satisfaction & project quality & $\begin{array}{c}\text { increase } \\
\text { of the resources } \\
\text { skill }\end{array}$ & $\begin{array}{c}\text { satisfaction } \\
\text { of the work team }\end{array}$ \\
\hline goal & company satisfaction & 0 & 0 & 0 & 0 \\
\hline $\begin{array}{c}\text { project quality } \\
\text { increase } \\
\text { project objectives }\end{array}$ & 0.701 & 0 & 0.667 & 0.250 \\
\cline { 2 - 6 } & $\begin{array}{c}\text { of the resources skill } \\
\text { satisfaction } \\
\text { of the work team }\end{array}$ & 0.193 & 0.667 & 0 & 0.750 \\
\hline
\end{tabular}

Table 15. Objective functions weights

\begin{tabular}{ccc}
\hline $\mathrm{Ob}_{1}$ & $\mathrm{Ob}_{2}$ & $\mathrm{Ob}_{3}$ \\
\hline 0.413 & 0.337 & 0.250 \\
\hline
\end{tabular}




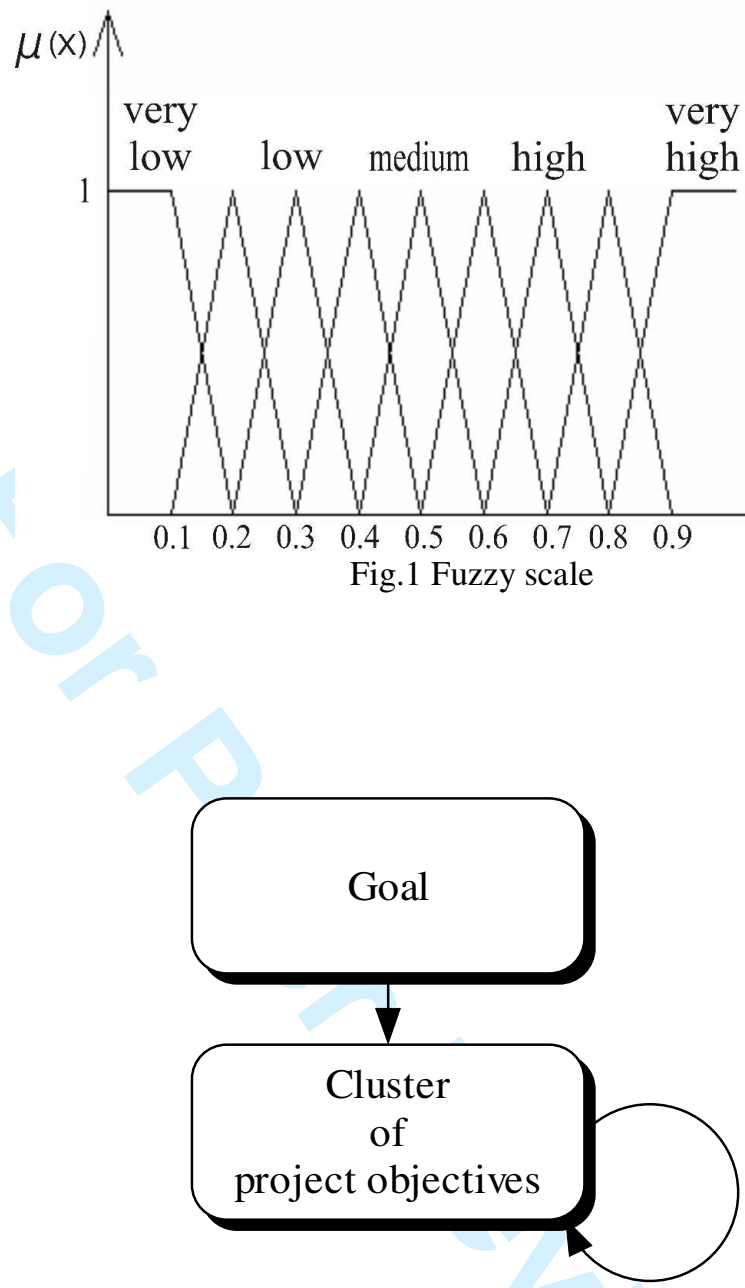

Fig.2 Network structure for objective functions weights assessment

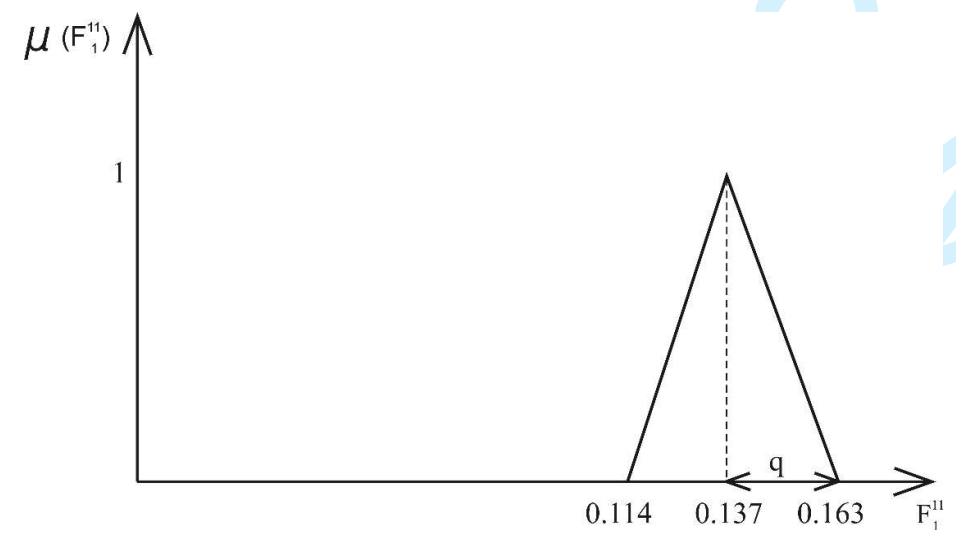

Fig.3 Fuzzy number representing $F_{1}$ of solution 11 
Table 16. Indifference threshold $q$

\begin{tabular}{cccc}
\hline solution & $F_{1}$ & $F_{2}$ & $F_{3}$ \\
\hline 1 & 0.030 & 0.541 & 0.260 \\
2 & 0.026 & 0.541 & 1.534 \\
3 & 0.029 & 0.516 & 2.080 \\
4 & 0.025 & 0.531 & 2.080 \\
5 & 0.034 & 0.512 & 0.130 \\
6 & 0.028 & 0.536 & 1.976 \\
7 & 0.027 & 0.540 & 1.794 \\
8 & 0.029 & 0.540 & 1.352 \\
9 & 0.029 & 0.536 & 1.872 \\
10 & 0.031 & 0.530 & 1.716 \\
11 & 0.026 & 0.537 & 2.028 \\
12 & 0.026 & 0.538 & 1.976 \\
13 & 0.033 & 0.522 & 0.572 \\
14 & 0.033 & 0.514 & 1.248 \\
15 & 0.034 & 0.519 & 0.572 \\
\hline
\end{tabular}

Table 17. Resources assignment for the solution 9

\begin{tabular}{c|ccccc}
\hline skill & 1 & 2 & 3 & 4 & 5 \\
\hline 1 & $6 ; 8$ & $4 ; 8$ & & $4 ; 5$ & \\
2 & & $4 ; 5$ & $1 ; 5$ & $2 ; 4$ & $1 ; 8$ \\
3 & $2 ; 3$ & & $1 ; 3$ & $2 ; 3$ & $1 ; 3$ \\
4 & $3 ; 6$ & $4 ; 5$ & $4 ; 5$ & $4 ; 5$ & $3 ; 6$ \\
5 & $3 ; 6$ & & $1 ; 3$ & $2 ; 3$ & $1 ; 6$ \\
\hline
\end{tabular}

Table 18 Resources assignment for the solution 10

\begin{tabular}{|c|c|c|c|c|c|}
\hline project & 1 & 2 & 3 & 4 & 5 \\
\hline 1 & $3 ; 8$ & $4 ; 5$ & & $4 ; 5$ & \\
\hline 2 & & $1 ; 5$ & $1 ; 5$ & 2 & $1 ; 5$ \\
\hline 3 & $3 ; 8$ & & $1 ; 3$ & 2 & $1 ; 6$ \\
\hline 4 & $6 ; 8$ & $6 ; 8$ & $4 ; 5$ & $4 ; 5$ & 6 \\
\hline 5 & 3 & & $4 ; 6$ & 2 & $1 ; 6$ \\
\hline
\end{tabular}

